\title{
Impacto na Qualidade de Vida de Pacientes com Câncer em meio à Pandemia de Covid-19: uma Reflexão a partir da Teoria das Necessidades Humanas Básicas de Abraham Maslow
}

doi: https://doi.org/10.32635/2176-9745.RBC.2020v66nTemaAtual.1068

\author{
Impact on the Quality of Life of Cancer Patients in the midst of the COVID-19 Pandemic: a Reflection from Abraham Maslow's \\ Theory of Basic Human Needs \\ Impacto en la Calidad de Vida de los Pacientes con Cáncer en Medio de la Pandemia Covid-19: un Reflejo de la Teoría de \\ Abraham Maslow sobre las Necesidades Humanas Básicas
}

Karine Marques Corrêa'; Júlia Damasceno Borges de Oliveira²; Gunnar Glauco de Cunto Carelli Taets ${ }^{3}$

\section{INTRODUÇÃO}

Segundo a Organização Mundial da Saúde (OMS) ${ }^{1}$, qualidade de vida é "a percepção do indivíduo de sua inserção na vida, no contexto da cultura e sistemas de valores nos quais ele vive e em relação aos seus objetivos, expectativas, padrões e preocupaçóes”. Entretanto, não é possível existir um conceito único e definitivo sobre qualidade de vida, mas se podem estabelecer elementos para pensar nessa noçáo em esferas objetivas e subjetivas A objetividade se refere à posição dos indivíduos e às suas relaçóes estabelecidas na sociedade. Já a subjetividade diz respeito ao conhecimento sobre as condiçôes físicas, emocionais, sociais e espirituais ${ }^{2}$ (biopsicossocioespiritual), o que é consonante com a definiçấo de saúde da OMS como "um estado de completo bem-estar físico, mental e social e não somente ausência de afecçôes e enfermidades". O paciente com câncer lida com alteraçôes em todos esses aspectos biopsicossocioespirituais. Sendo assim, o atual momento de pandemia mundial provocado pelo novo coronavírus (2019-nCoV) pode impactar na sua qualidade de vida.

Os coronavírus $(\mathrm{CoV})$ pertencem a uma grande família de vírus RNA fita simples que causam doenças desde um resfriado comum até doenças mais graves, como a síndrome respiratória aguda grave do coronavírus 2 (severe acute respiratory syndrome coronavirus 2 - Sars-CoV-2). Em de janeiro de 2020, cientistas chineses conseguiram isolar o Sars-CoV-2 em pacientes de Wuhan e, em 11 de fevereiro de 2020, a OMS nomeou a doença pelo coronavírus 2019 (coronavirus disease 2019) de Covid-193, que pode variar de um quadro clínico assintomático, sintomático de sintomas leves (febre, cansaço e tosse) a um quadro de sintomas graves (febre, alta, pneumonia e dispneia).

O grupo de risco para evolução com quadro clínico grave e morte por infecção por Covid-19 são pessoas com idade acima de 60 anos, portadores de doenças crônicas - asma, cardiopatias, diabetes, neoplasias - e imunodeprimidos. Logo, pacientes com câncer são um grupo de alto risco na pandemia da Covid-19, pois, além de já estarem vulneráveis à infecção por causa de sua doença subjacente, ainda estáo em estado imunossuprimido, aumentando o risco de desenvolver complicações biológicas graves do vírus ${ }^{4}$. Ademais, estão susceptíveis, como todos aqueles que enfrentam a pandemia e o isolamento social, a sentimentos como estresse, medo, angústia e solidão que causam prejuízo psicosocioespiritual.

Sendo assim, o cenário de pandemia e o isolamento social causado pela Covid-19 podem afetar diretamente a qualidade de vida dos pacientes com câncer nas dimensôes biopsicossocioespirituais. Nesse contexto, este texto reflete acerca dos impactos na qualidade de vida de pacientes com câncer, analisando os possíveis prejuízos de acordo com a teoria das necessidades humanas básicas de Maslow.

Este estudo, do tipo reflexivo com análise documental a partir de buscas realizadas na base de dados Google Acadêmico durante o mês de abril de 2020, foi pautado na teoria da motivação humana ou hierarquia das

${ }_{1}^{1}$ Acadêmica do curso de Medicina. Universidade Federal do Rio de Janeiro (UFRJ). Macaé (RJ), Brasil. Orcid iD: https://orcid.org/0000-0002-2801-9091

${ }^{2}$ Acadêmica do curso de Medicina. UFRJ. Macaé (RJ), Brasil. Orcid iD: https://orcid.org/0000-0002-6171-1944

${ }^{3}$ Enfermeiro. PhD em Ciências da Saúde. Professor-Adjunto da UFRJ. Macaé (RJ), Brasil. Orcid iD: https://orcid.org/0000-0003-4427-7864

Endereço para correspondência: Karine Marques Corrêa. Rua Almirante Raimundo Corrêa, 303, Bloco 2A, Apto. 904 - Bairro da Glória. Macaé (RJ), Brasil. CEP 27933-140. E-mail: correamkarine@gmail.com 
necessidades humanas básicas de Maslow5. Essa teoria organiza as necessidades básicas comuns a todos de forma hierárquica, sendo que a satisfação em um dado nível deve ser cumprida para se deslocar para o próximo nível, o que é uma motivação comportamental. Os níveis de necessidades criados por Maslow são cinco: (1) necessidades básicas e fisiológicas; (2) necessidades de segurança; (3) necessidades de amor; (4) necessidades de estima; e (5) necessidades de autorrealizaçáo.

\section{DESENVOLVIMENTO}

Os dados encontrados apontam quatro dimensóes para o impacto na qualidade de vida de pacientes com câncer no cenário de pandemia Covid-19, quais sejam: biológica, psicológica, social e espiritual. Nesse sentido, é possível traçar um paralelo da teoria das necessidades humanas básicas de Maslow com as quatro dimensōes da qualidade de vida de pacientes com câncer identificadas neste estudo: a dimensão biológica se enquadra nas necessidades fisiológicas; a psicológica permeia entre as necessidades fisiológicas, de amor e de autorrealização; a social se enquadra nas necessidades de amor; e a espiritual nas necessidades de segurança.

\section{DIMENSÃO BIOLÓGICA}

A dimensão biológica se enquadra nas necessidades fisiológicas definidas por Maslow, sendo a base da pirâmide. Isto implica que, sem saúde, o indivíduo não tem impulsos para buscar mais altos níveis de satisfação. Sendo assim, o paciente com câncer pode ter a dimensão biológical fisiológica afetada em diversos sentidos em razão da maior susceptibilidade de ser infectado pelo Sars-Cov-2 como resultado da imunossupressão; do possível atraso do tratamento e diagnóstico; e da alteração na rotina de dieta e exercícios físicos. Fatos que, somados, aumentam a incidência de sinais e sintomas, tal como dor, que reduzem substancialmente a qualidade de vida desse paciente ${ }^{4}$.

$\mathrm{O}$ primeiro estudo que explicitou a relação entre a Covid-19 e pacientes com câncer foi realizado na China em janeiro de 2020 e demonstrou que esses pacientes podem apresentar maior risco de pior prognóstico e morte por Covid-19, uma vez que, enquanto os eventos graves em pacientes não oncológicos foram de $8 \%$, o número em pacientes com câncer foi de $39 \%{ }^{6}$. Somado a esse estudo, uma análise comparativa entre pacientes oncológicos e não oncológicos ${ }^{7}$ mostrou que os pacientes com câncer são mais susceptíveis à infecção do que indivíduos sem câncer, por causa de seu estado imunossupressor sistêmico causado por tratamentos oncológicos, como quimioterapia ou cirurgia.

Com a medida de isolamento social para reduzir a disseminação da Covid-19, o acesso a práticas integrativas e complementares em saúde, tais como relaxamento e meditação, realizadas por pacientes com câncer, visando, especialmente, ao alívio de efeitos colaterais do tratamento ${ }^{8}$, está suspenso principalmente em regiōes endêmicas 9 . Neste caso, pode haver uma piora dos sintomas físicos relacionados ao câncer ou ao tratamento, afetando diretamente a qualidade de vida desse paciente.

Outro ponto a ser ressaltado na dimensão biológica é a alteraçáo nos hábitos alimentares durante o período de isolamento social. Alguns dos efeitos colaterais associados ao tratamento do câncer são anorexia, saciedade precoce, alteraçôes no olfato e paladar, além de problemas intestinais, culminando em uma piora nutricional dos pacientes ${ }^{10}$. Dessa forma, uma boa alimentação é essencial para a melhor qualidade de vida desse grupo. Entretanto, com a medida de isolamento atual, a compra de alimentos frescos e saudáveis é reduzida, enquanto práticas alimentares não saudáveis têm sido aplicadas.

A prática de exercícios físicos também é prejudicada no cenário atual, e estudos mostram que essa prática por pacientes com câncer promove benefícios como a redução do estado inflamatório crônico e da fadiga, a prevenção e tratamento da caquexia, além de maior adesão ao tratamento convencional e melhor qualidade de vida. Essas atividades devem ser individualizadas de acordo com o histórico e as limitaçôes e, se possível, feitas com acompanhamento profissional ${ }^{10,11}$. No entanto, durante a pandemia, as opçôes de atividades físicas são restritas ao ambiente doméstico, muitas vezes, sem o acompanhamento adequado, o que favorece o estilo de vida sedentário.

\section{DIMENSÃO PSICOLÓGICA}

De acordo com a teoria de Maslow, quando o indivíduo atinge, seja total ou parcialmente, suas necessidades, alcança níveis mais altos de saúde psicológica ${ }^{12}$, que está relacionada com a forma como uma pessoa reage às exigências, desafios e mudanças da vida, bem como o modo que harmoniza suas ideias e emoçôes. Logo, a dimensão psicológica, essencial à qualidade de vida, permeia entre as necessidades fisiológicas, de amor e autorrealização da hierarquia de Maslow.

Sendo assim, os impactos da quarentena podem ser percebidos por sintomas de transtorno de estresse pós-traumático (TEPT), transtornos de ansiedade e outros efeitos negativos desencadeados pelo tédio, estresse, medo, tempo de duração da quarentena e informações inadequadas sobre a doença ${ }^{13}$. Além disso, o estresse, por si só, pode levar a alterações imunológicas ${ }^{14} \mathrm{e}$, no padrão de sono $^{15}$, a fatores que causam impacto negativo na qualidade de vida desses pacientes.

Sabe-se, ainda, que o estresse, uma das consequências da pandemia, impacta negativamente na resposta imune 
do indivíduo. A resposta aos hormônios do estresse está associada à maior suscetibilidade a infecçóes e a doenças inflamatórias crônicas, autoimunes e alérgicas. Com isso, a ativação crônica do eixo neuroendócrino decorrente do estresse pode gerar o efeito imunossupressor por causa da liberação de glicocorticoides ${ }^{14}$. Quando se trata de pacientes com câncer, essa imunossupressão se torna ainda mais problemática, visto que o corpo já está debilitado em virtude da doença e do tratamento; com isso, o estresse é ainda mais prejudicial à saúde, afetando a qualidade de vida.

Além disso, outra consequência do estresse é a alteração no padrão de sono, que é essencial para saúde física e psicológica. Um estudo de revisão mostrou que as perturbaçôes do sono podem acarretar alteraçôes significativas no funcionamento físico, ocupacional, cognitivo e social do indivíduo, além de comprometer substancialmente a qualidade de vida ${ }^{15}$.

\section{DIMENSÃO SOCIAL}

Segundo Maslow ${ }^{5}$, manter relações sociais é uma necessidade básica humana de afeto e amor. Sendo assim, para realização pessoal, o ser humano precisa se relacionar com o meio e as pessoas que nele habitam, como terceira base hierárquica. Logo, no que tange à dimensão social da qualidade de vida de pacientes com câncer, observa-se uma dicotomia, uma vez que o isolamento social não pode ser aplicado na íntegra para esses pacientes, já que precisam continuar o tratamento, mas, simultaneamente, existe a necessidade de isolamento social de amigos e familiares.

Sabe-se que o tratamento oncológico demanda a interação com diversas áreas da saúde, o que envolve o contato com diversos profissionais em hospitais, clínicas e laboratórios. Isso significa que o isolamento social, se aplicado na íntegra para os pacientes oncológicos, resultará em prejuízos para o tratamento. Com isso, a recomendação de ficar em casa não pode ser seguida à risca por pacientes com câncer, o que aumenta o seu risco de contaminação ${ }^{16}$.

Por outro lado, o apoio de familiares e amigos, tão importante para o tratamento oncológico, é dificultado no cenário de isolamento social. O enfrentamento do câncer pode ser longo, cansativo, repleto de efeitos colaterais e, por isso, demanda reorganização familiar, apoio dos amigos e da comunidade ${ }^{17}$. Assim, passar pelo tratamento do câncer demanda atenção por parte dos familiares e amigos, fato que, durante o isolamento social, pode ser dificultado, pois nem todos têm acesso à Internet e à tecnologia, afetando diretamente a qualidade de vida e a necessidade básica de amor.

\section{DIMENSÃO ESPIRITUAL}

Uma possível interpretação para as necessidades de segurança de Maslow é a necessidade da espiritualidade ou de uma filosofia que organize o ser humano e o mundo onde habita de forma coerente e segura. Logo, Maslow considera o homem inseguro para escolher sozinho seus princípios e suas virtudes, necessitando de uma base para justificar seus atos, que, na maioria das vezes, é espiritual ou filosófica ${ }^{18}$.

Dessa forma, no que tange à dimensão espiritual, observou-se que há uma forte relação da fé como estratégia para o enfrentamento da doença ${ }^{19}$. Assim, as mudanças ocorridas na rotina da sociedade brasileira em geral, em decorrência da pandemia da Covid-19, podem gerar sentimentos de medo e desesperança.

Sendo assim, no contexto de qualidade de vida de pacientes com câncer, a espiritualidade promove suporte e força para lidar com as alteraçôes vivenciadas com a doença, como a dor, os efeitos colaterais do tratamento e a instabilidade emocional. Além disso, por meio da fé, muitos pacientes ressignificam a doença, ganhando força para enfrentar a situação, e se sentem estimulados para aderir ao tratamento convencional ${ }^{19}$. No entanto, o paciente, que está lidando com uma doença grave como o câncer, em um cenário de pandemia, sofre com o medo e a desesperança, podendo questionar a sua fé. A perda da segurança proporcionada pela espiritualidade pode ser um fator dificultador no enfrentamento do câncer, com consequente declínio na qualidade de vida desses pacientes.

\section{RECOMENDAÇ̃̃ES E REFLEXÕES PARA MINIMIZAR O IMPACTO NA QUALIDADE DE VIDA}

No cenário atual de pandemia causada pela Covid-19, pode-se esperar uma reduçáo na qualidade de vida de pacientes com câncer por declínio nas dimensôes biopsicossocioespirituais. No entanto, essa redução pode ser amenizada por meio de estratégias que já estão sendo elaboradas nos mais diversos centros de tratamento oncológico para o melhor manejo dos pacientes com câncer no contexto atual.

Em relação à manutenção do tratamento do câncer, há medidas que podem ser implementadas pelos centros de saúde: agendamento de consultas on-line ou por telefone para pacientes ambulatoriais, reduzindo o fluxo de pessoas; consultas feitas via Internet para orientaçôes sobre uso de medicamentos, manejo de sintomas e acompanhamento psicológico; substituição da quimioterapia intravenosa pela via oral, quando possível, reduzindo o risco para o paciente; aumento dos intervalos entre sessóes para quimioterapia adjuvante e redução do fracionamento na radioterapia, de acordo com as condiçóes do paciente, culminando em menor deslocamento; manter o rastreio da Covid-19 em profissionais da saúde e em pacientes com câncer; em casos mais graves de escassez de recursos 
em saúde, priorizar o acesso ao tratamento oncológico de acordo com a mortalidade associada ao não tratamento ${ }^{20,21}$.

Ademais, o paciente pode adotar novos hábitos em casa para melhorar a qualidade de vida durante o isolamento social: manter uma alimentação equilibrada, priorizando a compra de frutas e vegetais com maior durabilidade; praticar atividades físicas, respeitando os seus limites; criar uma rotina que garanta horas de sono restauradoras; se comunicar com familiares, amigos e grupos de apoio por telefone e via videochamadas; reduzir $\mathrm{o}$ acesso às notícias que podem causar estresse e ansiedade.

\section{CONCLUSÃO}

A reflexão aponta que é possível que exista dano na qualidade de vida de pacientes com câncer durante a pandemia de Covid-19. O maior impacto ocorre na dimensão biológica, como resultado do risco aumentado de complicações associadas à Covid-19, dificuldade de acesso ao tratamento, hábitos alimentares inadequados e sedentarismo, além de prejuízos à resposta imune do indivíduo.

Contudo, estratégias já vêm sendo traçadas pela comunidade científica da área da saúde para minimizar esses possíveis danos. Para que se possa cuidar melhor do paciente com câncer, sugere-se que sejam realizados estudos clínicos para melhor compreensão sobre os impactos da doença provocada pelo Sars-Cov-2, bem como meios de evitar a possível redução na qualidade de vida.

\section{CONTRIBUIÇÕES}

Todos os autores contribuíram igualmente na concepção e planejamento do estudo; na obtenção, análise e interpretação dos dados; na redação e revisão crítica, e aprovaram a versão final a ser publicada.

\section{DECLARAÇÃO DE CONFLITO DE INTERESSES}

Nada a declarar.

\section{FONTES DE FINANCIAMENTO}

Não há.

\section{REFERÊNCIAS}

1. The WHOQOL Group. The World Health Organization quality of life assessment (WHOQOL): position paper from the World Health Organization. Soc Sci Med. 1995;41(10):1403-9. doi: https://doi.org/10.1016/0277$9536(95) 00112-\mathrm{K}$
2. De Almeida MAB. Qualidade de vida e atividade física: explorando teoria e prática [Resenha]. Conexóes [Internet]. 2004 [acesso 2020 abr 10];2(2):105-8. doi: https://doi.org/10.20396/conex.v2i2.8637950 Resenha de: Gonçalves A, Vilarta R, organizadores. Qualidade de vida e atividade física: explorando teoria e prática. Barueri: Manole; 2004.

3. Cascella M, Rajnik M, Cuomo A, et al. Features, evaluation and treatment coronavirus (COVID-19) [updated 2020 Apr 6; cited 2020 Apr 10]. In: StatPearls [Internet]. Treasure Island (FL): StatPearls Publishing; 2020 Jan. Available from: https://www.ncbi.nlm.nih. gov/books/NBK554776/

4. The Lancet Oncology. COVID-19: global consequences for oncology [editorial]. Lancet Oncol. 2020;21(4):467. doi: https://doi.org/10.1016/S1470-2045(20)30175-3

5. Maslow AH. Uma teoria da motivação humana [Internet]. [1943] [acesso 2020 abr 14]. Disponível em: https://www. marciokarsten.pro.br/wp-content/uploads/2019/11/ Uma-teoria-da-motiva\%c3\%a7\%c3\%a3o-humana.pdf

6. Liang W, Guan W, Chen R, et al. Cancer patients in SARS-CoV-2 infection: a nationwide analysis in China. Lancet Oncol. 2020;21(3):P335-7. doi: http://doi. org/10.1016/S1470-2045(20)30096-6

7. Desai A, Sachdeva S, Parekh T, et al. COVID-19 and cancer: lessons from a pooled meta-analysis. JCO Glob Oncol. 2020;6:557-9. doi: http://doi.org/10.1200/ GO.20.00097

8. Cruz CT, Barros NF, Hoehne EL. Evidências sobre o uso de práticas alternativas e complementares no tratamento convencional de neoplasias mamárias. Rev Bras Cancerol. 2009;55(3):237-46.

9. Yang $G$, Zhang $H$, Yang Y. Challenges and countermeasures of integrative cancer therapy in the epidemic of COVID-19. Integr Cancer Ther. 2020;19:1534735420912811. doi: https://doi. org/10.1177/1534735420912811

10. Rock CL, Doyle C, Demark-Wahnefried W, el al. Nutrition and physical activity guidelines for cancer survivors. CA Cancer J Clin. 2012;62(4):242-74. doi: https://doi.org/10.3322/caac.21142

11. Nogueira HS, Lima WP. Câncer, sistema imunológico e exercício físico: uma revisão narrativa. Corpoconsciência [Internet]. 2018 [acesso 2020 abr 19];22(1):40-52. Disponível em: http://periodicoscientificos.ufmt.br/ojs/ index.php/corpoconsciencia/article/view/5636

12. Regis LFLV, Porto IS. A equipe de enfermagem e Maslow: (in)satisfaçôes no trabalho. Rev Bras Enferm. 2006;59(4):565-8. doi: http://dx.doi.org/10.1590/ S0034-71672006000400018

13. Zanon C, Dellazzana-Zanon LL, Weschler SM, et al. COVID-19: implicaçóes e aplicações da psicologia positiva em tempos de pandemia. Estud Psicol [Preprint]. 2020. doi:_https://doi.org/10.1590/SciELOPreprints.99 
14. Pagliarone AC, Sforcin JM. Estresse: revisão sobre seus efeitos no sistema imunológico. Biosaúde [Internet] 2009 [acesso 2020 abr 27];11(1):57-90. Disponível em: http:// www.uel.br/revistas/uel/index.php/biosaude/article/ view/24304

15. Müller MR, Guimarães SS. Impacto dos transtornos do sono sobre o funcionamento diário e a qualidade de vida. Estud Psicol. 2007;24(4):519-28. doi: http://dx.doi. org/10.1590/S0103-166X2007000400011

16. Kutikov A, Weinberg DS, Edelman MJ, et al. A war on two fronts: cancer care in the time of COVID-19. Ann Interno Med. 2020;172(11):756-8. doi: https://doi. org/10.7326/M20-1133

17. Ferreira NML, Dupas G, Costa DB, et al. Câncer e família: compreendendo os significados simbólicos. Cienc Cuid Saude. 2010;9(2):269-77. doi: https://doi. org/10.4025/cienccuidsaude.v9i2.8749

18. Ferreira A, Debute CM, Gimenez PEO. A teoria das necessidades de Maslow: a influência do nível educacional sobre a sua percepção no ambiente de trabalho. In: XIII Seminário em Administração; 2010 Set 9-10; São Paulo. Cid. Universitária (SP): PPGA- FEA-USP; 2010 [acesso 2020 abr 27]. Disponível em:_https://www.etica.eco.br/ sites/textos/teoria-de-maslow.pdf

19. Geronasso MCH, Coelho D. A influência da religiosidade/ espiritualidade na qualidade de vida das pessoas com câncer. Saúde Meio Ambient. 2012;1(1):173-87. doi: https://doi.org/10.24302/sma.v1i1.227

20. Wang H, Zhang L. Risk of COVID-19 for patients with cancer. Lancet Oncol. 2020;21(4):e181. doi: http://doi. org/10.1016/S1470-2045(20)30149-2

21. Hanna TP, Evans GA, Booth CM. Cancer, COVID-19 and the precautionary principle: prioritizing treatment during a global pandemic. Nat Rev Clin Oncol. 2020;17:268-70. doi: https://doi.org/10.1038/s41571020-0362-6 